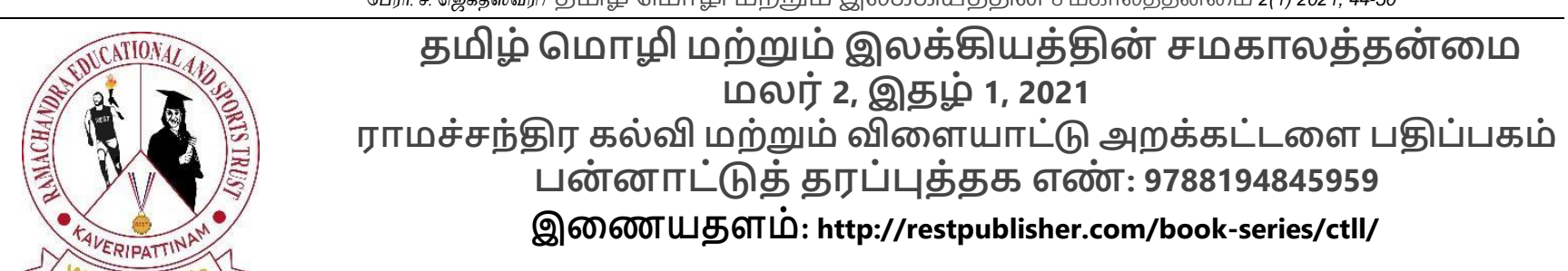

சங்க அக இலக்கியங்களில் களிறு(யானை) வழி அறியலாகும் இல்லற விழுமியங்கள்

பேரா. ச. ஜெகதீஸ்வரி

தமிழ்த்துறை (சுயநிதி), அருள் ஆனந்தர்கல்லூரி, கருமாத்தூர்.

\title{
ஆய்வுச் சுருக்கம்
}

இலக்கியம் என்பது நாம் வாழும் சமுதாயத்தைப் பிரதிபலிக்கும் கண்ணாடி ஆகும். சங்க இலக்கியம் என்பது சங்ககால மக்க்ளின் வாழ்வியல், சமூகம், பண்பாடு, கலாச்சாரம், முதலானவற்றை அறிய உதவுகின்றன.சங்க இலக்கியம் எட்டுத்தொகை, பத்துப்பாட்டு என்று பிரிக்கப்பட்டுள்ளது.ஏட்டுத்தொகை நூல்களில் எட்டு நூல்கள் உள்ளன.இந்த எட்டுநூல்களுள் அகம் குறித்த நூல்கள் ஐந்து ஆகும். எனவே புறச் செய்திகளை விட மனித வாழ்விற்குத் தேவையான அகவாழ்க்கைக்குப் புலவர்கள் முக்கியத்துவம் கொடுத்துள்ளதை உணர்ந்துகொள்ளலாம் அந்த அக வாழ்வு களவு, கற்பு என இரண்டாகப் பிரிக்கப்பட்டுள்ளன. திருமணத்திற்கு முந்தைய வாழ்வினைக் களவு வாழ்வு என்றும், திருமணத்திற்குப் பின் அமையும் வாழ்வினைக் கற்பு வாழ்வு என்றும் பிரித்துக் கூறியுள்ளனர். களவு, கற்பு என்னும் இவ்விருவகையான வாழ்க்கைப் படிநிலையில் தலைமக்களின் அன்பு நிலையை உணர்த்த அஃறிலை உயிர்களின் வாழ்க்கையைச் சுட்டிக்காட்டுவது சங்க இலக்கியம் பாடிய புலவர்களின் மரபு. மிக முக்கியமாகச் சங்க அக இலக்கியங்களான நற்றிணை, குறுந்தொகை, அகநானூறு, ஐங்குறுநூறு, கலித்தொகையில் விலங்குகளின் அன்பு நிலையைச் சுட்டி காதல், கற்பு வாழ்க்கையில் இருப்போர்க்கு வாழ்வியல் விழுமியங்களைக் கற்றுத்தர முற்பட்டதை அறியமுடிகிறது. இவ்விதமாக விலங்குகளின் காட்சிகளைச் சுட்டிக்காட்டி அவைகளின் அன்பு நிலையை விளக்குவதும், அதன் வழித் தலைமக்களுக்கு அன்பின் ஆழத்தை உணர்த்துவதும்தான் புலவர்களின் நோக்கமாகவும் இருந்திருக்கிறது. அந்த வகையில் சங்க அக இலக்கியங்களில் அஃறிணை விலங்குகளில் ஒன்றான யானையின் வழி இல்லற விழுமியமாகக் கருதப்படும் குடும்பத்தைக் காத்தல் என்னும் பண்பு ஆய்வுக்கு உட்படுத்தப்பட்டுள்ளது . பி.எல்.சாமி எழுதிய சங்க இலக்கியத்தில் விலங்கின விளக்கம் என்னும் நூலில் உள்ள கருத்துக்களின் அடிப்படையில் இவ்வாய்வுக்கட்டுரை அணுகப்பட்டுள்ளது .

முன்னுரை

சங்ககாலம் முதற்கொண்டு இக்காலம் வரை மனிதன் தான் வாழ்கின்ற சுற்றுப்புறச் சூழலையும், சுற்றுப்புறத்தால் வாழ்கின்ற உயிரினங்களையும் சார்ந்தே வாழ்கின்றான். அவ்வாறு சார்ந்து வாழ்கின்றபோது சுற்றுப்புறமும் சுற்றுப்புறத்தில் வாழ்கின்ற உயிரினங்களும் மனிதனுக்குப் பல்வேறு நிலையில் துணை நிற்கின்றன. அவ்வாறே மனிதனும் சுற்றுப்புறத்திற்கும், சுற்றுப்புறத்தில் வாழ்கின்ற உயிரினங்களுக்கும் பாதுகாப்பாக இருந்துவருகிறான். இவ்வாறு ஒன்றை ஒன்று சார்ந்து வாழ்கின்ற சூழ்நிலையிலும் கூட மனிதனுக்கு அவனைச் சார்ந்து வாழ்கின்ற உயிரினங்களின் வாழ்க்கை, அவனது வாழ்க்கையைச் செம்மைப்படுத்திக்கொள்ள துணை நிற்கின்றன. சான்றாக,

“எறும்பினைப் போல் சுறுசுறுப்பாக இரு”; “காக்கையைப் போல் ஒற்றுமையாக இரு” “நாயைப் போல் நன்றியோடு இரு" என்பதைக் கூறலாம். இவ்உயிரினங்கள் மனிதர்களுக்குப் பெரிதும் பயன்படுபவை அல்ல. எனினும் அவைகள் மக்களின் வாழ்க்கைக்குப் படிப்பினையாக உள்ளன .

மனிதனைச் சுற்றி வாழ்கின்ற விலங்குகளின் வாழ்க்கை முறையினைப் புலவர்கள் தாங்கள் படைத்த படைப்புகளில் வெளிப்படுத்தி, மனித வாழ்க்கையைச் செம்மைப்படுத்தியிருக்கின்றனர். அவ்வகையில், யானையின் வாழ்க்கையின் வழியாக மனித சமூகத்திற்கு என்ன வகையான வாழ்க்கைச் சிந்தனைகளை உணர்த்துகிறார்கள் என்று 
சில சங்க அகப் பாடல்களின் வழி அறியலாம்.

தமிழ் நாட்டில் யானையை விடப் பெரிய விலங்கு இல்லை என்று கூறலாம். யானையின் தனித்த சிறந்த உறுப்புகளுள் தந்தமெனப்படும் மருப்பும், துதிக்கையுமாகும். ஆண்யானைக்கே தந்தங்கள் இருந்ததாக விலங்கு நூலார் கூறுவர். யானையின் தந்தம் பால்நிற வெண்மையானதென்பதை 'பால் மருப்பு' என்று கலித்தொகை கூறும். இதகை,

“மாசில் வெண்கோட்டு அண்ணல் யானை"(அகம்.251.) என்றும் கூறப்பட்டுள்ளது. தும்பிக்கை பற்றி,

“ஒய்களி றெடுத்த நோயுடை நெடுங்கை

தொகுசொற் கோடியர் தூம்பி னுயிர்க்கும்"(அகம்.111.) என்று கூறப்பட்டுள்ளது. ‘தடக்கை'(நற்.317) என்றும் கூறப்பட்டுள்ளது.

யானையின் கால்களை தூணுக்கு ஒப்பிட்டுக் கூறுவதை “களிற்றுத் தாள் புரையுந் திரிமரப்பந்தர்”(பொரு.187) என்று கூறப்பட்டள்ளது,

யானையின் காலடிகளை 'பாவடி'(அகம்.211) என்றும், யானையின் தலையை “நிலஞ்செலச் செல்லாக் கயந்தலைக் குழவி"(நற்.171) என்றும் கூறப்பட்டுள்ளது .

\section{யானையின் வாழிடம்}

"யானைகள் குன்றுகளும் மலைகளும் சூழ்ந்த அடர்நத ஈரம் நிறைந்த காடுகளில் காணப்படுகின்றன. சங்க இலக்கியத்தில் வரும் செய்திகள் பெரும்பாலும் காட்டானையைப் பற்றியவையே. தமிழ்நாட்டில் யானைகள் நிறையக் காணப்படுகின்றன. சாலைகளிலும் காணப்படுகின்றன. முக்கியமாக மூங்கில் காடுகள் நிறைந்த குன்றுகளிலும் மலைகளிலும் யானைகள் விரும்பி வாழும்.

யானை வாழுமிடங்களாக “வாழையஞ் சிலம்பு“(அகம்.332) “துன்னருஞ் சோலை“ (குறுந்.83), “வாழை யோங்கிய தாழ்கண சும்பு“(அகம்.8), “கழை திரங்கடுக்கம்"(அகம்.347), “பனியிருஞ் சோலை“(அகம்.119), “மலை முதல் அடுக்கம்“(அகம்.148), நெடுங்கழைநிவந்த நிழல்படு சிலம்பு“(நற்.392), “குன்றத்துக் கவாஅன்“(அகம்.202), என்று சங்க அக இலக்கியங்கள் கூறுகின்றன"1.

\section{யானையின் செயற்பாடுகளும் பண்புகளும்}

"யானைகள் வெயிற்காலத்தில் வறட்சியினால் வெகுதொலைவு நீரைத் தேடி அலையும் என்று அறிஞர்கள் கூறியுள்ளனர். யானைகள் நீர்நிலையில் படிந்து திளைக்கும் செய்தியையும் கூறியுள்ளனர். யானை படிந்த நீர்நிலை சேறும் சகதியுமாக மாறித் தோன்றும். யானைகள் கூட்டமாகக் கூடி நீர்நிலைக்குச் செல்லுங்கால் கன்றுகள், பெண் யானைகள் முதலில் நீரில் படிந்து விளையாடிய பிறகு ஆண்யானைகள் நீரில் இறங்குவதுண்டு என்று கூறுவர். வறட்சியுற்ற காலத்தில் ஆண்யானையானது கொம்பால் வறண்டுபோன ஆற்று மணலைத் தோண்டி நீரை ஊறும்படி செய்தும் குடிப்பதுண்டு"2(நற்.240,நற்.186)

"யானைகள் கூட்டமாக நீர் நிலைகளுக்குச் செல்லுங்கால் முதலில் யானைக்கன்றுகளைக் குளிக்க விடும். வறட்சியான காலத்தில் கன்றுகளும் பிடிகளும் நீர் குடித்துக் குளித்த பின் ஆண்யானைகள் காத்திருந்து எஞ்சிய நீரைக் குடிக்கும். ஆண்யானையானது கல்லூற்றில் கிடைத்த சிறிது நீரைத் தும்பிக்கையால் உறிஞ்சித் தன்னுடைய துடை பெண்யானைக்குக் கொடுக்க ஓடியதாகக் கூறப்பட்டுள்ளது"3.(நற்.186)

ஆண்யானையின் தனிப்பழக்கத்தை மிக அழகாக கலித்தொகையில் (பாடல் 11) கூறப்பட்டுள்ளது. மேலே அறிஞர்கள் கூறியது போல் யானைக் குட்டிகள் குளித்துக் கலக்கிய நீரைப் பெண்யானை குடித்த பிறகு ஆண்யானை பின்னர் குடித்ததாகக் கூறிய செய்தி சங்கப் புலவர்கள் நேரில் கண்டு கூறிய அரிய செய்தியாகும்"4.

யானையின் உணவுப் பழக்கம்

"யானையின் உணவுப் பழக்கத்தைப் பற்றியும் சங்க நூல்களில் பல செய்திகள் கூறப்பட்டுள்ளன. யானை பெரும்பாலும் காட்டில் மரம், செடி, கொடிகளின் தழைகளை, இலைகளைப் பறித்துத் தின்னும். துதிக்கையால் இகைக் கொம்புகளைக் கொடிகளை வளைத்து வாயில் போடுவதால் யானை உண்ணும் இம்முறையைக் “கவளம்" என்று சங்க நூல்களில் அழைத்தனர். பின்னர் யானையின் உணவே கவளம் என்று பெயர் பெற்றது"5.(அகம்.157, குறுந்.170)

"யானை, விளாங்கனியையும் சோலை வாழையையும் பலாவின் பழத்தையும் உண்ணும் பழக்கமுடையது"6. 
"ஆண்யானையின் முக்கிய செயற்பாடிகளில் ஒன்று தன் துணையைக் காத்தல் என்பது · தன் கடமையிலிருந்து தவறாத ஆண்யானையின் செயற்பாட்டினை குறுந்தொகை(255)யில் கூறப்பட்டுள்ளது. அதாவது, பொத்தல் இல்லாத வைரம் பாய்ந்த யாமரத்தை தன் கொம்பால் குத்தியதாகக் கூறப்பட்டுள்ளது . பொத்தல் இருந்தால் அது ஏற்கனவே சாறு எடுக்கப்பட்ட மரம். பொத்தல் இல்லாதிருந்தால் அது சாறு உள்ள மரம் என்று யானைக்குத் தெரிந்திருந்தது. நற்றிணையில் (92) யாமரத்தை சினம்கொண்டு குத்தி அதில் கிடைத்த நீரை தன் பெண்யானைக்குக் கொடுத்ததாகச் செய்திகள் கிடைக்கின்றன"7.

\section{யானையின் காதல் வாழ்வு}

"யானையின் காதல் வாழ்வை ஆராய்ந்து சங்கப் புலவர்கள் எழுதியுள்ளனர். ஆண்யானை பெண்யானையுடன் இருக்கும்போது எப்போதும் சேர்ந்தே கானப்படும். ஆண்யானையும் பெண்யானையும் துதிக்கையைத் தழுவி இன்புறும்(குறுந்.332). பெண்யானைக்கு ஆண்யானை விருப்பமான உணவை எடுத்துக்கொடுத்து அன்பை வெளிப்படுத்தும்(அகம்.218). அமர்துணை தழீஇ(குறுந்.215) என்றெல்லாம் செய்திகள் காணக்கிடைக்கின்றன. அதனாலேயே தலைவன் தலைவிக்கு அன்பு காட்டுவதை ஆண்யானை பெண்யானைக்கு அன்பு பாராட்டுவதுடன் ஒப்பிட்டுச் சங்க நூல்கள் கூறுகின்றன"8.

\section{கலித்தொகை - யானைக் குடிம்பம்}

கலித்தொகையில் பாலைக்கலியில் தலைவனது பிரிவை எண்ணிக் கலங்காது மனதைரியம் கொண்டவளாக மனஉறுதியோடு தலைவி தோழியிடம் கூறும்பொழுது, தனது மனதைாியத்திற்கும் மனஉறுதிக்கும் காரணம் பாலை நிலத்தில் வாழும் பல காட்சிகளில் ஒன்றான யானை குடும்பத்துடன் வாழும் காட்சியே என்று எடித்துரைக்கின்றாள். பாலைத்திணையில் பாலை பாடிய பெருங்கடுங்கோ பாடிய அப்பாடல், “தலைவி மூன்றன் பகுதி தலைவன் கூறிப் பொருள்வயிற் பிரிகின்ற காலத்து காடு கடியவாயினும், இவ்வகைப் பட்டனவும் உள என்று கூறினார். அவை காண்டலின் வருவர் எனத் தோழிக்குக் கூறி அதற்கு நிமித்தமும் கூறி ஆற்றுவித்தது“ என்னும் தலைவி கூற்றாக அமைந்துள்ளது .

அப்பாடலில், “அறவழியில் வாழக் கருதும் தலைவன், தலைவியின் உள்ளம் ஏற்கும் வண்ணம் நல்லுரைகள் பல கூறி, பாலை நிலம் கடந்து பொருள் தேட் சென்றான். அவனது போக்கைக் கண்டு தோழி பெரிதும் வருந்தினாள்.தலைவனது உட்கருத்தை நன்குணர்ந்த தலைவி தோழிக்கு ஆறுதல் கூறுகின்றாள்.

முதலாவதாக, தலைவனின் நன்நெறிகளையும், ஒழுக்கத்தைக் கடைப்பிடிக்கும் தன்மையும், ஆன்றோர் காட்டிய பாதையைக் கடவாத அருளுள்ளம் கொண்டவர் என்றும், பொருளின் பயனை நன்கு கண்டறிந்தவர் என்றும் பொருள் ஒன்றினால்தான் அறஞ்செய்யக் கூடும், இன்பம் நுகர்தல் கூடும் என்பதை நன்கு ஆய்ந்த பின்னரே பொருள் தேடச் சென்றிருக்கின்றார் என்று தோழியிடம் கூறி, பின் நம்மை விட்டுப் பிரிந்து சென்ற தலைவன் விரைவில் வருவார் என்பதற்குண்டான காரணங்கள் சிலவற்றைக் கூறுகிறேன் கேட்பாயாக! என்று கூறத் தொடங்கினாள்.

பொருளுக்காகப் பிரிந்து செல்வதற்கு முன் பாலை நிலத்தின் கொடிய வெம்மைத் தன்மையை என் நெஞ்சம் அஞ்சும் வண்ணம் எடுத்துரைத்தார்.அந்நிலத்திலும் அருளோடு வாழும் உயிர்களும் இல்லாமல் இல்லை என்பதையும் என் பேதையுள்ளம் பெரிதும் விரும்புமாறு விளக்கியுரைத்தார். ஈருடலும் ஓரயிருமாய் வாழ்ந்த தலைவர் நம்மை விட்டுப் பிரிந்தவர் மீண்டும் வாரார் என்று எண்ணுவது நம் அறியாமையன்றி வேறில்லை என்பதைத் தன் கருத்தின் முடிவாகக் கூறுவதற்குக் காரணமான காட்சியை,

“அரிதாய அறனெய்தி யருளியோர்க் களித்தலும்

பெரிதாய பகைவென்று பேணாரைத் தெறுதலும்

புரிமவமர் காதலின் புணர்ச்சியும் தருமெனப்

பிரிவெண்ணிப் பொருள்வயிற் சென்றநங் காதலர்

வருவார்கொல் வயங்கிழாஅய் வலிப்பல்யான் கேளினி

அடிதாங்கும் அளவின்றி யழலன்ன வெம்மையால்

கடியவே கனங்குழாஅய் காடென்றார் அக்காட்டுள்

துடியடிக் கயந்தலை கலக்கிய சின்னீரைப் 
பிடியுட்டிப் பின்னுண்னுங் களிறெனவும் உரைத்தனரே'

இனைநல முடைய கானம் சென்றோர் "9 (கலி.பாலை..11) என்று கலித்தொகையில் கூறப்பட்டுள்ளது .

அப்பாடலில், நெருப்பைப் பரப்பி வைக்கப்பட்ட இடம் எப்படி வெம்மை மிகுந்து காணப்படுமோ, அத்தகைய நிலையையுடையது பாலைநிலம். அந்நிலத்தில் வாழும் உயிரினங்களும் வெம்மையைத் தாங்காமல் துன்புறும் தன்மையுடையது. அடி வைத்தால் பொறுக்க முடியாத அளவு வெம்மை நிறைந்து காணப்படும். அந்த அளவுக்கு வெம்மை நிறைந்ததாயினும் அக்காட்டில் நீரை விரும்பிய களிறு, பிடி, கன்று ஆகிய மூன்றும் சுற்றித் திரிந்தன.அலைந்து திரிந்து இறுதியில் ஓர் சிறிய நீர் நிலையைக் கண்டுபிடித்தன.

அவை தங்களுக்கே உரிய பழக்கமான செயலைச் செய்தன. அதாவது, வறட்சியான காலத்தில் நீர்நிலைகளைக் கண்டால் முதலில் யானைக் கன்று அச்சிறிய நீரில் இறங்கி விளையாடி பின்பு நீர் அருந்தி, பின்பு பிடியும், அதன் பின்பே ஆண்யானையான களிறும் நீர் உண்னுதல் பழக்கமாம். இப்பழக்கத்தை அப்படியே பின்பற்றி மகிழ்ச்சியாக வாழ்ந்தன. இதனை

"துடியடிக் கயந்தலை கலக்கிய சின்னீரைப்

பிடியுட்டிப் பின்னுண்னுங் களிறெனவும் உரைத்தனரே'(கலித்.11) என்னும் வரிகள் புலப்படுத்தி நிற்கின்றன.

கொடுமை வாய்ந்த பாலை நிலத்தில் ஐந்தறிவு படைத்த யானைக் குடும்பத்தின் அன்பு நிறைந்த காட்சியும் உண்டென்று தலைவன் தன்னிடம் முன்னமே கூறியிருந்ததாகத் தோழியிடம் தலைவி கூறினாள்.

மேலும் அவர் இனிய நிழலே எங்குமே காணமுடியாத அளவிற்கு வற்றிப் போன மரங்களே மிகுதியாகக் காட்சியளிக்கும் தன்மையுடையது பாலைநிலம். நிலத்துக்கடியில் நெடுந் தொலைவு வேர்விட்டு நீரை உறிஞ்சும் வண்மை வாய்ந்தவை மரங்கள்.அம்மரங்களும் நீரின்றி வாடுகின்றதென்றால் அப்பாலை நிலத்தின் நிலையை என்னென்று எடுத்துரைப்பது? மரங்களும் நீரின்றி வற்றிய நிலையிலேயே பெரிதும் வெம்மை மிகுந்தது அப்பாலைநிலம் என்றார். ஆனால் , அக்காட்டிலும் அன்புடன் வாழும் உயிரினங்கள் இல்லாமல் இல்லை என்றும் கூறினார்.

அவ்விலங்குகளைக் கண்ட நம் தலைவர் நம்முள்ளமும் உடலும் வலியும் பொலிவும் அற்றுப்போகும் அளவிற்கு நம்மைக் கைசோர விடவேமாட்டடார் என்பதை தோழிக்கு உணர்த்தினாள்.

\section{குறுந்தொகை - யானை குடிம்பக் காட்சி}

குறுந்தொகையில், பாலை நிலத்தில் வாழும் ஆண்யானை தன் பெண்யானையின் பசியினைப் போக்குவற்காக யாமரத்தின் பட்டையை உரித்தாகப் பாடலொன்று உள்ளது. பாலை பாடிய பெருங்கடுங்கோ பாடிய அப்பாடல், ‘தோழி, ‘கடிது வருவார்' என்று ஆற்றுவித்தது’ என்னும் கூற்றாக அமைந்துள்ளது.

அதாவது.தோழி தலைவியிடம், “தோழியே! தலைவர் நின்னிடம் மிக்க விருப்பம் உடையவர்.அவர் நின்மாட்டுத் தலையளி செய்தலும் உடையர். அவர் நின்னைப் பிரிந்து சென்ற நெறிகள், பெண் யாயையின் பசியை நீக்குவதற்காகப் பெரிய கையையுடைய ஆண்யானையானது, மெல்லிய கிளைகளையுடைய யா மரத்தின் பட்டையை உரித்து, அதன் நீரை அப்பிடி பருகச் செய்யும் அன்பைப் புலப்படுத்தற்கு இடமாக உள்ளன" என்று கூறினாள். இதனை,

“நசை பரிது உடையர்; நல்கலும் நல்குவர்;

பிடிபசி களைஇய பெருங்கை வேழம்

மென்சினை யாஅம் பொளிக்கும்

அன்பின் தோழி - அவர் சென்ற ஆறே."10 (குறுந்.37)

என்று காட்சிப்படுத்தியுள்ளார் புலவர். இக்காட்சியின் வழி தலைவனின் அன்பை தலைவி தோழிக்கு உணர்த்துகிறாள் .

தலைவியைப் பிரிந்து செல்வதற்கு முன்பே, தலைவி மாட்டு அன்புடைய காதலர், அன்புடைய காட்சிகளைக் கண்டவழி பேரன்புடையராவர் எனத் தலைவி உணரும்படியாக உரைத்தாள் என்பதை புலவர் உணரச்செய்கிறார்.

ஆண்யானை தன் பசியைப் போக்கிக்கொள்ள நினையாமல், தன்னுடைய பிடியின் பசியைப்போக்க முயலுதலைக் கண்ட தலைவன், முன்னைவிட பேரன்புடையனாய்த் திரும்புதல் நிச்சயம் என்னும் பொருள்பட தோழி 
கூறியதாக உணரமுடிகிறது.

இடைச்சுரத்தின் கண் அன்பை வெளிப்படுத்தும் காட்சிகளைக் கண்டு, வினை முடித்து, விரைந்து வந்து, தன் மனை திரும்பித் தலைவியை இன்புறுத்துவான் என்று தோழியின் கூற்றிலிருந்து உணர்ந்துகொள்ளலாம்.

\section{அகநானூறு - யானைக் குடும்பக் காட்சி}

பொருட்செல்வத்தின் அருமையை உணர்ந்தவனும் அதேசமயம் தன் தலைவியின் தன்மையை உணர்ந்தவனுமான தலைவனை பொருளீட்டவேண்டும் என்று இடைவிடாது வற்புறுத்திய நெஞ்சை நோக்கி தலைவன் கூறுவதாக அமைந்தது அப்பாடல், “தலைமகன் பொருள் கடைக்கூட்டிய நெஞ்சினைக் கழறிச் செலவு இடித்துரைத்தது“ என்னும் கூற்றாக மதுரைத் தத்தங்கண்ணனார் பாடிய அப்பாடலில், பொருட்செல்வத்தின் அருமை கூறி தலைவியைப் பிரிந்து பொருள்தேட வேண்டுமென்று இடைவிடாது வற்புறுத்தியது அவனது மனம். ஆனால் தலைவனோ, தலைவியைப் பிரிந்து செல்வதற்கு மனமில்லை. அதுமட்டுமல்ல, தலைவி இல்லாது அந்த அருங் சுரத்தைக் கடத்தல் என்பது அரிது. ஆனால் தலைவி தன்னுடன் வந்தாலோ அந்த அருஞ்சுரத்தை கடந்துவிடலாம். ஆனால் தான் செல்லக் கூடிய நீரற்ற அந்த பாலை நிலத்தில் நீர்தே அலைந்த யானை தன் பெண் யானைக்கு நீர் தருவதற்காக யாமரத்தின் அடியைத் தன் கொல்லும் கொம்பு ஒடியுமாறு குத்தி உலர்ந்த கிளைகளையுடைய அம்மரத்தினது பட்டையைக் கிழித்துத் துன்பம் தருகின்ற வேனிற் காலத்தில் தான் இன்பம் பெறுகின்ற தன் பிடியை உண்ணும்படி செய்யும் . இத்தகைய அருஞ் சுரத்து வழி மென்மையான தலைவியை அழைத்துக்கொண்டு செல்வதென்பது மிகவும் அரிது என்று தனக்குள் சொல்லிக்கொண்டான்.

“யானும் அறிவென் மன்னே; யானைதன்

கொல்மருப்பு ஒடியக் குத்தி, சினம்சிறந்து

இன்னா வேனில் இன்துணை ஆர,

முளிசினை மரா அத்துப் பொளிபிளந்த ஊட்ட,

புலம்புவீற் றிருந்த நிலம்பகு வெஞ்சுரம்

அரிய அல்லமன், நமக்கே" 11(அகம்.335:4-9)

என்று அகநானூற்றுப் பாடலிலும் காணலாம்.

இப்பாடலில், அன்பு நிறைந்த யானைக் குடும்பம் வறட்சியான சூழலில் வாழும் வாழ்வை நினைத்துப் பார்த்த தலைவன் அவ்யானைக் குடும்பம்போல தானும் மகிழ்ச்சியாக வாழவேண்டும் என்ற எண்ணம் உடையவனாக இருந்தான். அதுமட்டுமல்ல, வறட்சியான பாலை நிலத்தின் வழி தன் தலைவியை அழைத்துச் செல்வதென்பது அவளுக்கு எத்தகைய இடையூறினை ஏற்படுத்தியிருக்கும் என்பதையும் புரிந்தவனாக இருந்தான் என்பதை புலவர் உணர்த்துகிறார்.

\section{நற்றிணை - யானைக் குடிம்பக் காட்சி}

தலைவியைப் பிரிந்த தலைவன் தன்னை மறந்தாரோ என்று ஐயுறுகிறாள். அப்பொழுது அவளது தோழி அவளை ஆற்றுவிக்கிறாள். அவள் அவ்வாறு ஆற்றுவிப்பதற்கு அவள் எடுத்துக்கொண்ட காட்சி பாலை நிலத்தில் அன்பான யானைக் குடும்பக் காட்சியாகும். இக்காட்சி “பிரிவிடை வேறுபட்ட கிழத்திற்குத் தோழி சொல்லியது" என்னும் கூற்றாகப் பாடப்பட்டுள்ளது. இக்கருத்து,

"உள்ளார்கொல்லோ-தோழி- துணையொடு

வேனில் ஓதி பாடு நடை வழலை

வரி மரல் நுகும்பின் வாடி, அவண

வறன் பொருந்து குன்றத்து உச்சி கவாஅன்

வேட்டச் சீறூர் அகன் கண் கேணிப்

பய நிரைக்கு எடுத்த மணி நீர்ப் பத்தார

புன் தலை மடப் பிடிகன்றோடு ஆர

வில் கடிந்து ஊட்டின பெயரும்

கொல் களிற்று ஒருத்தல் சுரன் இறந்தோரே"12 (நற்.92) 
என்று பதிவுசெய்யப்பட்டுள்ளது.

தோழி தலைவியிடம் கூறும்பொழுது, “பனிமூட்டம் போன்ற பொய்நீராகிய மரல்நீரே அங்குத் தென்படும் நீர். ஓந்தியும் பாம்பும் வாடிக் கிடக்கும் வழியில் அவர் சென்றுள்ளார். அங்கே வறண்ட குன்றத்து உச்சி மலைப் பிளவில் வேட்டுவர் வாழும் சிற்றூரில் ஆடுமாடுகள் நீர் உண்ண அகலமான நீர்க்கேணி தோண்டி வைத்திருப்பர். அது நீலநிற நீரைக் கொண்ட பத்தர் என்னும் குழிக்கேணி. அந்தக் கேணிப்பத்தர் அருகில் வேட்டுவர் கொடிய விலங்குகளை வேட்டையாட வில்லுப்பொறி அமைத்திருப்பர். அந்த வில்லுப்பொறியைத் தகர்த்த ஆண்யானை தன் பெண்யானைக்கும் கன்றுக்கும் அந்தப் பத்தர்கேணியில் நீரூட்டி அழைத்துச் செல்லும். இப்படிப்பட்ட பாலைநிலக் காட்டு வழியில் தலைவர் சென்றிருக்கிறார். அவ்வாறு செல்லும்போது நம்மை நினைக்கமாட்டார் போல் இருக்கிறது“ என்று கூறினாள்.

இந்தக் காட்சியின் வழி தோழி சில உண்மைநிலைகளைத் தலைவிக்குப் புலப்படுத்தினாள். அதாவது அன்புநிறைந்த யானைக் குடும்பத்தினைக் கண்டும் தலைவியினை விரைந்து சென்று காணவேண்டும் என்ற எண்ணமில்லாமல் காலம் தாழ்த்தும் தலைவனை எண்ணி வருந்தி என் பயன் என்று தலைவனின் பொறுப்பின்மையை உணர்த்தினாள்.

ஐங்குறுநூறு - யானைக் குடும்பக் காட்சி

\section{தொகுப்புரை}

தலைவி தன்னைப் பிரிந்துசென்ற தலைவன் தான் மேற்கொண்ட வினையை முடித்துக்கொண்டு விரைவில் திரும்பி வருவான் என்ற மனஉறுதி ஏற்படுவதற்கு வறண்ட பாலை நிலத்திலும் அன்புள்ளம்கொண்டு வாழும் யானைக்குடும்பக் காட்சியே காரணமாக அமைந்தது.

பிடியின் பசியைப் போக்க ஆண்யானை மேற்கொண்ட செயல் தலைவியைப் பேணிக் காக்க வேண்டும் என்ற இல்லறக் கடமையினை தலைவனுக்கு நினைவூட்டி நிற்பதை அறிந்துகொள்ளலாம்.

பொருள் தேடி தலைவியைப் பிரிந்து செல்ல வேண்டிய சூழலில் தண்ணீரற்ற வறண்ட நிலத்தில் வாழும் ஆண்யானையின் செயற்பாட்டினைக் கருத்தில் கொண்டு கடுமையான அப்பாலை வழியில் மென்மைத் தன்மை வாய்ந்த தன் தலைவியை உடன் அழைத்துச் செல்வதென்பது இயலாத காரியம், அதேசமயம் தலைவியைப் பிரிந்து தனியே அப்பாலை நிலத்தில் தான் கடத்தல் என்பதும் அரிதான காரியம் என்று தனக்குள் சொல்லிக்கொணட தலைவனின் நிலை தலைவியின் மீது மிகுந்த அக்கறை கொண்டவன் என்பதைப் புலப்படுத்துகிறது .

ஆண்யானை தன் கன்றையும் பிடியையும் நீர் உண்பிக்கின்ற காட்சியை கண்டபின்னும் தலைவன் காலம் தாழ்த்தினான் என்பது அவனது குடும்பப் பொறுப்பின்மையை உணர்த்துகிறது.

முடிவுரை

ஆறறிவு படைத்த மனிதன் ஐந்தறிவு முதல் ஓரறிவு வரை உள்ள விலங்குகள் , பறவைகள், இயற்கைகள் முதலானவற்றிலிருந்து சில படிப்பினைகளைப் பெறுகிறான். அவை காலந்தோறும் மனித வாழ்வு மேம்பட உதவுவனவாக உள்ளன. அதனடிப்படையில் யானையின் வாழ்விலிருந்து புலவர் பெருமக்கள் கற்றுணர்ந்த செய்தியினை மனித வாழ்வு மேம்பட, மிகமுக்கியமாக குடிம்பம் என்கிற அமைப்பு மேம்பட தாங்கள் படைத்த பாடல்களில் பதிவு செய்து வைத்துள்ளனர் என்பதை இவ்வாய்வுக்கட்டுரையின் முடிவாகக் கொள்ளலாம்.

\section{அடிக்குறிப்பு}

1. பி.எல்.சாமி, சங்க இலக்கியத்தில் விலங்கின விளக்கம், ப.277

2. மேலது, ப.295.

3. மேலது, ப.297.

4. மேலது, ப.297.

5. மேலது, ப.301.

6. மேலது, ப.310.

7. மேலது, ப.315.

8. மேலது, ப.338. 
9. முனை.அ. விசுவநாதன்(உ.ஆ.), கலித்தொகை(மூ.உ.), ப.38

10. முனை.வி.நாகராசன்(உ.ஆ.), குறுந்தொகை(மூ.உ.), ப.103.

11. முனை. இரா. செயபால்(உ.ஆ.),அகநானூறு(மூ.உ.), ப.984.

12. நற்றியை

\section{ஆய்வுக்கு உட்படுத்திய நூல்கள்}

1.கலித்தொகை

2.அகநானூறு

3. நற்றிணை

4. குறுந்தொகை

5. சங்க இலக்கியத்தில் விலங்கின விளக்கம்
நியூ செஞ்சுரி புக் ஹவுஸ்

அம்பத்தூர்

சென்னை - 2004

நியூ செஞ்சுரி புக் ஹவுஸ்

அம்பத்தூர்

சென்னை - 2004

நியூ செஞ்சுரி புக் ஹவுஸ்

அம்பத்தூர்

சென்னை - 2004

நியூ செஞ்சுரி புக் ஹவுஸ்

அம்பத்தூர்

சென்னை - 2004

திருநெல்வேலித் தென்னிந்திய

சைவசிந்தாந்த நூற்பதிப்புக்

கழகம், சென்னை -1

முதற்பதிப்பு 1970 